\title{
Effect of Tea Extracts and Phenolic Components on Immunoglobulin Production by Mesenteric Lymph Node Lymphocytes of Sprague-Dawley Rats
}

\author{
Koji Yamada, Takashi Watanabe, Shihoko Kaku, Nasra Hassan and Michihiro Sugano \\ Laboratory of Food Science, Department of Food Science and Technology, Faculty of Agriculture, Kyushu University 6-10-1, \\ Hakozaki, Higashi-ku, Fukuoka 812-81, Japan
}

Received September 26, 1996

\begin{abstract}
We examined the effect of tea extracts on immunoglobulin (Ig) production of mesenteric lymph node lymphocytes of Sprague-Dawley rats and found that various extracts suppressed the production of IgA, IgG, and IgM. In addition, the original extracts of green and black teas strongly enhanced IgE production, and their $10^{2}$ or $10^{3}$ times diluted samples exerted an inhibitory effect. Similarly, tea polyphenols having a triphenol group enhanced IgE production at $1 \mathrm{mM}$ and inhibited it at concentrations below $100 \mu \mathrm{M}$. On the contrary, diphenolic epicatechin did not affect IgE production. Though all tea polyphenols exerted inhibitory effect on the production of IgA and IgG at concentrations above $10 \mu \mathrm{M}$, triphenolic compounds, such as epigallocatechin gallate, gallic acid and pyrogallol, exerted an enhancing tendency on IgA production at $0.1 \mu \mathrm{M}$. These results suggest that the Ig production-regulating activity of tea extract is partly due to tea polyphenols, especially those having a triphenol group.
\end{abstract}

Keywords: tea extract, tea polyphenols, EGCg, IgE antibody, IgA antibody, food allergy

Among four types of allergic reactions, type I allergy plays an important role in the incidence of allergies against food components and airborne antigens (Metcalfe, 1991). In the type I allergy, induction of allergen-specific immunoglobulin $\mathrm{E}(\mathrm{IgE})$ is an essential step for the initiation of allergic reaction, while allergen-specific $\operatorname{IgA}$ inhibits the allergic reaction through the inhibition of allergen absorption in the gut (Metcalfe, 1991). Thus, class-specific regulation of $\mathrm{Ig}$ production, the inhibition of $\operatorname{lgE}$ production and the stimulation of IgA production may alleviate allergic reactions. Such class-specific regulation of $\mathrm{Ig}$ production is induced by lymphokines, such as IL-4, IL-5, IFN- $\alpha$, and IFN- $\gamma$ (Pene $e t$ al., 1988; Gauchat et al., 1991; Ochel et al., 1991; Rousset et al., 1991; Elson \& Beagley, 1994). In addition, several biomaterials, such as bile acids, lectins, and unsaturated fatty acids, exert a class-specific regulation of Ig production of rat mesenteric lymph node (MLN) lymphocytes (Yamada et al., 1993, 1996; Lim et al., 1994a, b). MLN lymphocytes belong to the gut immune system and inhibit allergen absorption through the production and secretion of allergen-specific IgA. Thus, the study on the interaction between food components and MLN lymphocytes is important for the prevention of food allergy.

It has been reported that the consumption of green tea reduced the risk of esophageal cancer (Gao et al., 1994) and atherosclerosis (Stensvold et al., 1992). Among green tea components, tea polyphenols (TP) have been reported to have anti-viral (Nakayama et al, 1991) and anti-tumor (Fujita et al., 1989; Agarwal et al., 1992; Taniguchi et al, 1992) activities. In addition, they exert an anti-allergic effect through the inhibition of chemical mediator release from rat peritoneal exudate cells (Matsuo et al., 1996). Green tea is produced from the leaves of Camellia sinensis, as well as black, oolong and puaru teas, and TP content varies with the method of manufacturing (Yen \& Chen, 1996). In addition to these teas, various types of teas prepared from other plants such as tochu and gymnema are also consumed. To clarify their effects on allergy incidence, we examined here the Ig production-regulating activity of various tea extracts and TP using rat MLN lymphocytes.

\section{Materials and Methods}

Materials Tea leaves prepared from Camellia sinensis (green, black, oolong, and puaru) and other plants, such as Eucommia ulmoides (tochu), Gymnema sylvestre (gymnema), Houttuynia cordata (dokudami), Diospyros kaki (kakinoha), and Equisetum arvense (sugina) were obtained from the local market. One hundred $\mathrm{ml}$ of boiled water was poured into $10 \mathrm{~g}$ of tea leaves and stood for $30 \mathrm{~min}$ at room temperature. The extracts were then filtered through two sheets of gauze, and the filtrates were centrifuged at $8,000 \times g$ for $20 \mathrm{~min}$. (-)-Epicatechin (EC), (-)-epicatechin gallate $(\mathrm{ECg}),(-)$-epigallocatechin (EGC), and (-)-epigallocatechin gallate $(\mathrm{EGCg})$ were purchased from Kurita Water Industries (Tokyo), and catechol, resorcinol, hydroquinone, pyrogallol, and gallic acid from Sigma (St. Louis, MO, USA). These polyphenols were dissolved in a phosphate-buffered saline, $\mathrm{pH} 7.4$ (PBS). All of these sample solutions were sterilized through a $0.22 \mu \mathrm{m}$ nitrocellulose filter (Millipore, Bedford, MA, USA) and diluted with sterilized PBS.

Cells and cell culture MLN lymphocytes were prepared from 9- to 10-week-old male Sprague-Dawley rats and cultured as previously described (Lim et al., 1994b). In short, MLN was excised from the rats under light diethyl ether anesthesia, and lymphocytes were isolated using LympholyteRat (Cedarlane, Hornby, Canada). The cells were cultured in $800 \mu 1$ of RPMI 1640 medium, $100 \mu 1$ of fetal bovine serum (Intergen Co., NY, USA) and $100 \mu \mathrm{l}$ of sample solution in a 
24-well culture plate (Becton Dickinson, Franklin Lakes, NJ, USA) for $4 \mathrm{~h}$ to determine $\operatorname{IgE}$ and for $24 \mathrm{~h}$ to determine other Igs. The Ig content of the culture supernatant was measured by the enzyme-linked immunosorbent assay (ELISA), as previously described (Lim et al, 1994a, b). Data obtained from multiplicated cultures were expressed as mean $\pm \mathrm{SE}$, and the difference was analyzed using Student's $t$-test.

\section{Results}

Ig production-regulating activity of tea extracts First, the dose-dependent effect of various tea extracts on Ig production by MLN lymphocytes was examined. As shown in Fig. 1, the original solution of green tea extract strongly enhanced IgE production by 6.5 -fold. A similar enhancement was observed in the original solutions of black (5.1- fold), puaru (3.2-fold), dokudami (2.3-fold), and oolong (2.2-fold) teas. However, inhibition of IgE production was observed in diluted samples, especially in green and black tea extracts. The inhibitory effect was maximal in $10^{2}$ times diluted sample of green tea (93\% inhibition) and $10^{3}$ times diluted sample of black tea ( $89 \%$ inhibition).

In the case of $\operatorname{IgA}$ production, inhibitory activity was observed in 10 or $10^{2}$ times diluted extracts of green, black, oolong, and puaru teas. The inhibition of IgA production was moderate in the extracts of tochu and gymnema teas, and the effect of dokudami, kakinoha, and sugina teas was negligible.

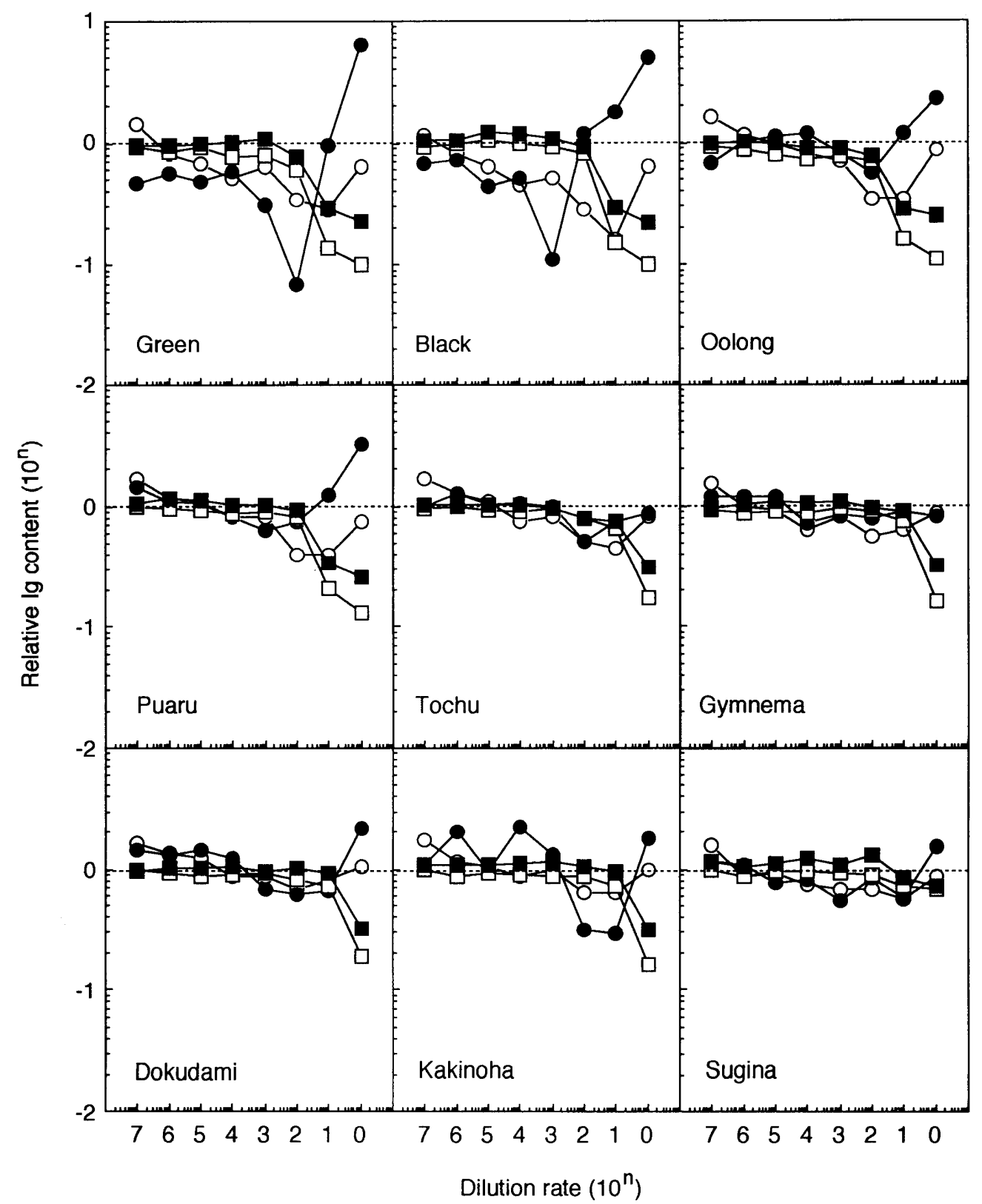

Fig. 1. Effect of tea extracts on immunoglobulin production by MLN lymphocytes of Sprague-Dawley rats. MLN lymphocytes were cultured in the presence of various concentrations of tea extracts, and the concentrations of $\operatorname{IgA}(O), \operatorname{IgE}(\bullet), \operatorname{IgG}(\square)$, and $\operatorname{IgM}(\boldsymbol{\square})$ in culture supernatants were determined by ELISA. Dotted lines show the control Ig levels without tea extracts. 

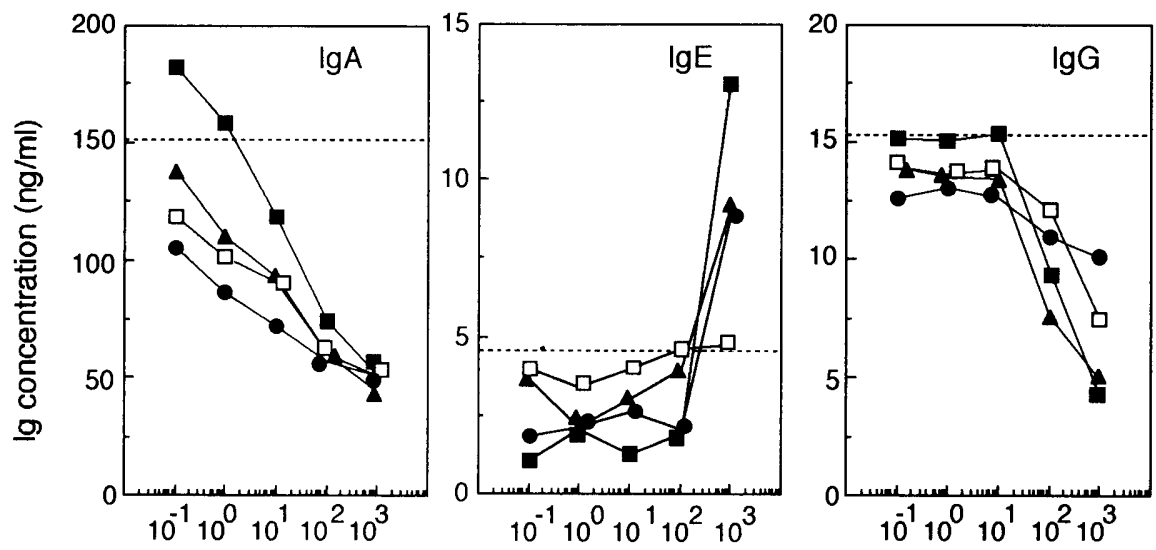

Polyphenol concentration $(\mu \mathrm{M})$

Fig. 2. Dose-dependent effect of tea polyphenols on immunoglobulin production by MLN lymphocytes of Sprague-Dawley rats. MLN lymphocytes were cultured

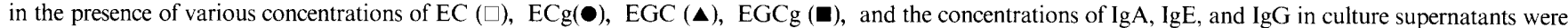
determined by ELISA. Dotted lines show the control Ig levels without tea polyphenols.

Table 1. Effect of tea polyphenols on immunoglobulin production by MLN lymphocytes.

\begin{tabular}{lcccl}
\hline Additives & IgA $(\mathrm{ng} / \mathrm{ml})$ & IgE $(\mathrm{ng} / \mathrm{ml})$ & IgG $(\mathrm{ng} / \mathrm{ml})$ & IgM $(\mathrm{ng} / \mathrm{ml})$ \\
\hline None & $34.9 \pm 2.3$ & $11.4 \pm 0.6$ & $10.5 \pm 0.2$ & $3.51 \pm 0.11$ \\
EC & $28.2 \pm 2.3$ & $10.3 \pm 0.9$ & $9.4 \pm 0.1^{* *}$ & $2.81 \pm 0.10^{* *}$ \\
ECg & $37.8 \pm 2.5$ & $9.6 \pm 0.7$ & $9.4 \pm 0.2^{* *}$ & $3.28 \pm 0.19$ \\
EGC & $36.4 \pm 4.1$ & $10.7 \pm 0.8$ & $9.9 \pm 0.1^{*}$ & $3.42 \pm 0.03$ \\
EGCg & $45.2 \pm 4.1^{*}$ & $8.4 \pm 0.7^{* *}$ & $11.2 \pm 0.3$ & $3.79 \pm 0.14$ \\
\hline
\end{tabular}

MLN lymphocytes were cultured with $0.1 \mu \mathrm{M} \mathrm{TP}$, and Ig content of culture supernatants was determined by ELISA. Data are mean \pm SE of 4 cultures, and difference is considered to be significant at ${ }^{*} p<0.05$ and ${ }^{* *} p<0.01$.

In the case of IgG and IgM production, the inhibitory effect was observed in the original and 10 times diluted extracts of green, black, oolong, and puaru teas and in original extracts of tochu, gymnema, dokudami, and kakinoha teas, while it was negligible in the sugina tea extract. These results suggest that tea leaves prepared from Camellia contain Ig productionregulating components more abundantly than tea leaves produced from other plants.

Ig production-regulating activity of polyphenol compounds In search of active components in the tea extracts, the Ig production-regulating activity of $\mathrm{TP}$ was examined. As shown in Fig. 2, all TP exerted dose-dependent inhibition of IgA production as in the case of tea extracts. Among them, EGCg solely exerted weak IgA production-enhancing tendency at $0.1 \mu \mathrm{M}$, but inhibited it at concentrations above 10 $\mu \mathrm{M}$. On the other hand, IgE production was strongly enhanced with EGCg and moderately with EGC and ECg at 1 $\mathrm{mM}$, while it was weakly inhibited at concentrations below $100 \mu \mathrm{M}$. In contrast to the above triphenolic compounds, the EC with a diphenol group did not affect IgE production. However, all TP inhibited IgG production at concentrations above $100 \mu \mathrm{M}$.

To show the effect of TP on the Ig production of the lymphocytes at the physiological level, the cells were treated with $0.1 \mu \mathrm{M}$ TP. As shown in Table 1, EGCg significantly
Table 2. Effect of polyphenols on immunoglobulin production by spleen lymphocytes.

\begin{tabular}{lcccc}
\hline Additives & $\mathrm{IgA}(\mathrm{ng} / \mathrm{ml})$ & $\mathrm{IgE}(\mathrm{ng} / \mathrm{ml})$ & $\mathrm{IgG}(\mathrm{ng} / \mathrm{ml})$ & $\mathrm{IgM}(\mathrm{ng} / \mathrm{ml})$ \\
\hline None & $13.8 \pm 0.6$ & $6.49 \pm 0.32$ & $10.4 \pm 0.2$ & $4.25 \pm 0.07$ \\
Catechol & $15.4 \pm 2.1$ & $5.41 \pm 0.45$ & $10.0 \pm 0.3$ & $4.45 \pm 0.08$ \\
Resorcinol & $13.5 \pm 1.3$ & $5.90 \pm 0.23$ & $9.7 \pm 0.1^{* *}$ & $4.27 \pm 0.05$ \\
Hydroquinone & $14.9 \pm 0.9$ & $6.68 \pm 0.43$ & $9.9 \pm 0.2$ & $4.27 \pm 0.15$ \\
Pyrogallol & $19.4 \pm 2.3^{*}$ & $5.91 \pm 0.62$ & $10.1 \pm 0.2$ & $4.11 \pm 0.18$ \\
Gallic acid & $20.3 \pm 2.1^{*}$ & $7.22 \pm 0.70$ & $9.8 \pm 0.1^{*}$ & $4.09 \pm 0.11$ \\
EGCg & $20.6 \pm 2.6^{*}$ & $4.49 \pm 0.36^{* *}$ & $10.8 \pm 0.3$ & $4.24 \pm 0.20$ \\
\hline
\end{tabular}

MLN lymphocytes were cultured with $0.1 \mu \mathrm{M}$ of phenolic compounds, and Ig content of culture supernatants was determined by ELISA. Data are mean \pm SE of 5 cultures, and difference is considered to be significant at ${ }^{*} p<0.05$ and ${ }^{* *} p<$ 0.01

enhanced IgA production and inhibited IgE production. In addition, EC, ECg, and EGC inhibited IgG production, and EC inhibited IgM production.

To examine the contribution of the triphenol structure on the Ig production-regulating activity of TP, the cells were treated with $0.1 \mu \mathrm{M}$ of various polyphenols. As shown in Table 2, triphenolic pyrogallol and gallic acid exerted $\operatorname{IgA}$ production-enhancing tendency similar to $\mathrm{EGCg}$, while diphenolic catechol, resorcinol, and hydroquinone did not. Among them, EGCg solely inhibited the IgE production of the lymphocytes. These results suggest that the triphenol structure is important for the stimulation of IgA production, but some other factor(s) participated in the inhibition of IgE production.

\section{Discussion}

Epidemiological results suggest that green tea consumption reduces the risk of esophageal cancer (Gao et al., 1994) and atherosclerosis (Stensvold et al., 1992). We demonstrated here that the extracts prepared from tea leaves prepared from Camellia, especially green and black tea extracts, exerted strong Ig production-regulating activity. These extracts exerted a bifunctional effect on IgE production: stimulative effect 
in the original extracts and inhibitory effect in the diluted extracts. In addition, inhibition of IgA production was induced by the extracts of green, black, oolong, and puaru teas. However, the effect of the extracts prepared from the tea leaves of other plants on $\operatorname{IgE}$ and $\operatorname{IgA}$ production was much weaker than that of the above Camellia tea extracts.

TP are major constituents of green tea extract and exert various physiological effects, such as anti-viral (Nakayama et al., 1991) and anti-cancer (Fujita et al., 1989; Agarwal et al, 1992; Taniguchi et al., 1992; Gao et al., 1994) activities. In addition, they exert anti-allergic activity by inhibiting the release of chemical mediators such as histamine and leukotriene $\mathrm{B}_{4}\left(\mathrm{LTB}_{4}\right)$ from rat peritoneal exudate cells (Matsuo et al., 1996). Because $\mathrm{LTB}_{4}$ stimulates the $\mathrm{IgE}$ production of $\mathrm{B}$ lymphocytes by enhancing the expression and release of soluble CD23 (Yamaoka et al., 1989; Dugas et al., 1990), the inhibition of $\mathrm{LTB}_{4}$ production by $\mathrm{TP}$ may affect the $\mathrm{IgE}$ productivity of $\mathrm{B}$ cells.

Thus, we examined the effect of TP on Ig production by rat MLN lymphocytes and found that TP exert a similar Ig production-regulating activity as seen in green and black tea extracts. All TP inhibited the production of $\operatorname{IgA}$ and $\mathrm{IgG}$, as well as the above tea extracts, while the bifunctional effect on IgE production was observed only in TP with a triphenol group. In addition to $\mathrm{ECg}, \mathrm{EGC}$, and $\mathrm{EGCg}$, triphenolic pyrogallol and gallic acid enhanced IgA production at 0.1 $\mu \mathrm{M}$, but diphenolic compounds did not. These results suggest that TP are responsible for the expression of the Ig production-regulating activity of green and black tea extracts and that the triphenol group plays a crucial role. Similarly, transformed cell-specific toxicity (Mitsui et al., 1995) and chemical mediator release-inhibiting activity (Matsuo et al, 1996) were observed only in catechins having the triphenol structure. Thus, the triphenol interacts with cell components more strongly than the diphenol.

$\mathrm{EGCg}$ is the most abundant component of green tea extract, representing 40-60\% of TP (Graham, 1992) and exerts the strongest biological activities (Mitsui et al, 1995; Matsuo et al., 1996). It exerts an anti-allergic effect by inhibiting the release of histamine and $\mathrm{LTB}_{4}$ from rat peritoneal exudate cells at a concentration of $100 \mu \mathrm{M}$ (Matsuo et al., 1996). In addition, we showed here that EGCg inhibits IgE production and enhances $\operatorname{IgA}$ production of MLN lymphocytes at 0.1 $\mu \mathrm{M}$. Orally administered catechins are detected in the serum, liver, lung, and skin of rats (Hackett \& Griffiths, 1983) and also in the serum, urine, and feces of humans (Das, 1971). When $(+)$ catechin was orally administered to men at a dose of $92.3 \mathrm{mg} / \mathrm{kg}$, the increase in blood phenol level was 4 to 21 $\mu \mathrm{g} / \mathrm{ml}$ (corresponding to $14-72 \mu \mathrm{M}$ ) during $48 \mathrm{~h}$ after the single administration (Das, 1971). Thus, there is a possibility that tea consumption reduces the risk of allergy, as reported in cancer (Gao et al., 1994) and atherosclerosis (Stensvold et al., 1992). However, the TP content of Camellia tea extracts varies with the method of manufacturing, 26.7\% in green, $23.2 \%$ in oolong and $4.3 \%$ in black teas (Yen \& Chen, 1996), and the Ig production-regulating activity of tea extracts did not completely coincide with their TP content. This suggests that tea extracts contain Ig production-enhancing or suppressing factors other than TP.
Acknowledgments This work was partly supported by the Special Coordination Funds for Promoting Science and Technology of the Science and Technology Agency of Japan, and by the Fund from The San-Ei Gen Foundation for Food Chemical Research.

\section{References}

Agarwal, R., Katiyar, S.K., Zaidi, S.I.A. and Mukhtar, H. (1992). Inhibition of skin tumor promoter-caused induction of epidermal ornithine decarboxylase in SENCAR mice by polyphenolic fraction isolated from green tea and its individual epicatechin derivative. Cancer Res., 52, 3582-3588.

Das, N.P. (1971). Studies on flavonoid metabolism. Biochem. Pharmacol., 20, 3435-3445.

Dugas, B., Paul-Eugene, N., Cairns, J., Gordon, J., Calenda, A., Mencia-Huerta, J.M. and Braquet, P. (1990). Leukotriene $B_{4}$ potentiates the expression and release of $\mathrm{Fc} \varepsilon \mathrm{RII} / \mathrm{CD} 23$, and proliferation and differentiation of human $\mathrm{B}$ lymphocytes induced by IL-4. J. Immunol., 145, 3406-3411.

Elson, C.O. and Beagley, K.W. (1994). Cytokines and immune mediators. In "Physiology of the Gastrointestinal Tract," ed. by L.R. Johnson. Raven Press, New York, pp. 243-265.

Fujita. Y., Yamane, T., Tanaka. M., Kuwata, K., Okuzumi, J., Takahashi, T., Fujiki, H. and Okuda, T. (1989). Inhibitory effect of (-)-epigallocatechin gallate on carcinogenesis with $n$-ethyl- $n^{\prime}$ nitro- $n$-nitrosoguanidine in mouse duodenum. Jpn. J. Cancer Res., 80, 503-505.

Gao, Y.T., Mclaughlin, J.K. and Blot, W.J. (1994). Reduced risk of esophageal cancer associated with green tea consumption. J. Natl. Cancer Inst. 86, 855-858.

Gauchat, J.F., Gascan, H., Roncarolo, M.G., Rousset, F., Pene, J. and de Vries, J.E. (1991). Regulation of human IgE synthesis: the role of $\mathrm{CD}^{4+}$ and $\mathrm{CD}^{8+} \mathrm{T}$-cells and the inhibitory effects of interferon- $\alpha$. Eur. Respir. J., 4, 31s-38s.

Graham, H.N. (1992). Green tea composition, consumption, and polyphenol chemistry. Prev. Med., 21, 334-350.

Hackett, A.M. and Griffiths, L.A. (1983). The disposition of 3-Omethyl-(+)-catechin in the rat and marmoset following oral administration. Eur. J. Drug Metab. Pharmacokinet., 8, 35-42.

Lim, B.O., Yamada, K. and Sugano, M. (1994a). Inhibition of immunoglobulin production in human Namalwa cells and rat spleen lymphocytes by bile acids. Biosci. Biotech. Biochem., 58 , $1107-1111$.

Lim, B.O., Yamada, K. and Sugano, M. (1994b). Effect of bile acids and lectins on immunoglobulin production in rat mesenteric lymph node lymphocytes. In Vitro Cell. Dev. Biol., 30A, 407-413.

Matsuo, N., Yamada, K., Yamashita, K., Shoji, K., Mori, M. and Sugano, M. (1996). Inhibitory effect of tea polyphenols on histamine and leukotriene $\mathrm{B}_{4}$ release from rat peritoneal exudate cells. In Vitro Cell. Dev. Biol., 32, 340-344.

Metcalfe, D.D. (1991). Food allergy. Current Opinion Immunol, 3, 881-886.

Mitsui, T., Yamada, K., Yamashita, K., Matsuo, N., Okuda, A., Kimura, G. and Sugano, M. (1995). E1 A-3Y1 cell-specific toxicity of tea polyphenols and their killing mechanism. Int. J. Oncol., 6, 377-383.

Nakayama, M., Toda, M., Okubo, S. and Shimamura, T. (1991). Inhibition of influenza virus infection by tea. Lett. Appl. Microbiol., 11, 38-40.

Ochel, M., Vohr, H-W., Pfeiffer, C. and Gleichmann, E. (1991). IL-4 is required for the IgE and IgGl increase and IgGl antoantibody formation in mice treated with mercuric chloride. J. Immunol., 146 , 3006-3011.

Pene, J., Rousset, F., Briere, F., Chretien, I., Wideman, J., Bonnefoy, J.Y. and De Vries, J.E. (1988). Interleukin 5 enhances interleukin 4 -induced IgE production by normal human B cells. The role of soluble CD23 antigen. Eur. J. Immunol, 18, 929-935.

Rousset, F., Robert, J., Andary, M., Bonnin, J.P., Souillet, G., Chretien, I., Briere, F., Pene, J. and De Vries, J.E. (1991). Shift in interleukin- 4 and interferon- $\gamma$ production by $T$ cells of patients with elevated serum $\operatorname{lgE}$ levels and the modulatory effects of these lymphokines on spontaneous $\mathrm{IgE}$ synthesis. J. Allergy Clin. Im- 
munol., 87, 58-69.

Stensvold, I., Tverdal, A., Solvoll, K. and Foss, O.P. (1992). Tea consumption. Relationship to cholesterol, blood pressure, and coronary and total mortality. Prev. Med., 21, 546-553.

Taniguchi, S., Fujiki, H., Kobayashi, H., Go, H., Miyado, K., Sadano, H. and Shimokawa, R. (1992). Effect of (-)-epigallocatechin gallate, the main constituent of green tea, on lung metastasis with mouse B16 melanoma cell lines. Cancer Lett., 65, 51-54.

Yamada, K., Lim, B.O. and Sugano, M. (1993). Suppression of immunoglobulin production of human Namalwa cells and rat lymphocytes by bile acids. In Vitro Cell. Dev. Biol., 29A, 840-841.
Yamada, K., Hung, P., Yoshimura, K., Taniguchi, S., Lim, B.O. and Sugano, M. (1996). Effect of unsaturated fatty acids and antioxidants on immunoglobulin production by mesenteric lymph node lymphocytes of Sprague-Dawley rats. J. Biochem., 120 (1), 138-144.

Yamaoka, K.A., Claesson, H.E. and Rosen, A. (1989). Leukotriene $B_{4}$ enhances activation, proliferation, and differentiation of human $\mathrm{B}$ lymphocytes. J. Immunol., 143, 1996-2000.

Yen, G.-C. and Chen, H.-Y. (1996). Relationship between antimutagenic activity and major components of various teas. Mutagenesis, 11, 37-41. 